\title{
Magnetic Reconnection as a Driver for a Sub-ion-scale Cascade in Plasma Turbulence
}

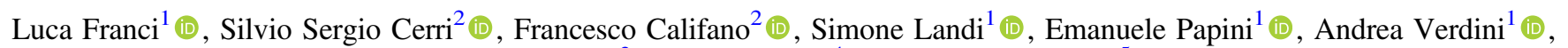 \\ Lorenzo Matteini $^{3}$ (D), Frank Jenko ${ }^{4}$ (D), and Petr Hellinger ${ }^{5}$ (D) \\ ${ }^{1}$ Department of Physics and Astronomy, University of Florence, Via G. Sansone 1, I-50019 Sesto F.no, Firenze, Italy \\ ${ }^{2}$ Physics Department "E. Fermi," University of Pisa, Largo B. Pontecorvo 3, I-56127 Pisa, Italy \\ ${ }^{3}$ Department of Physics, Imperial College London, London SW7 2AZ, UK \\ ${ }^{4}$ Max Planck Institute for Plasma Physics, Boltzmannstr. 2, D-85748 Garching, Germany \\ 5 Astronomical Institute, CAS, Bocni II/1401, CZ-14100 Prague, Czech Republic \\ Received 2017 July 19; revised 2017 September 22; accepted 2017 October 15; published 2017 November 17
}

\begin{abstract}
A new path for the generation of a sub-ion-scale cascade in collisionless space and astrophysical plasma turbulence, triggered by magnetic reconnection, is uncovered by means of high-resolution two-dimensional hybridkinetic simulations employing two complementary approaches, Lagrangian and Eulerian, and different driving mechanisms. The simulation results provide clear numerical evidence that the development of power-law energy spectra below the so-called ion break occurs as soon as the first magnetic reconnection events take place, regardless of the actual state of the turbulent cascade at MHD scales. In both simulations, the reconnection-mediated smallscale energy spectrum of parallel magnetic fluctuations exhibits a very stable spectral slope of $\sim-2.8$, whether or not a large-scale turbulent cascade has already fully developed. Once a quasi-stationary turbulent state is achieved, the spectrum of the total magnetic fluctuations settles toward a spectral index of $-5 / 3$ in the MHD range and of $\sim-3$ at sub-ion scales.
\end{abstract}

Key words: magnetic reconnection - solar wind - turbulence

Supporting material: animations

\section{Introduction}

Turbulent dynamics and its interplay with magnetic reconnection in collisionless plasmas is of great interest in many different astrophysical environments, e.g., in the interstellar medium, in accretions disks, and in stellar coronae and winds. Direct in situ measurements of near-Earth turbulent plasmas, such as the solar wind and the terrestrial magnetosheath, have led to increasingly accurate constraints on the turbulent energy spectra and on the magnetic field structure (Bruno \& Carbone 2013; Stawarz et al. 2016; Matteini et al. 2017). These observations determine the typical spectral slopes for turbulent electromagnetic fluctuations and reveal the presence of a break in the power spectra at ion kinetic scales (Bale et al. 2005; Alexandrova et al. 2009; Chen et al. 2010; Sahraoui et al. 2010), separating a magnetohydrodynamic (MHD) inertial cascade from a kinetic cascade. The former is generally characterized by a $-5 / 3$ slope in the magnetic power spectrum, whereas the latter is quite steeper, with a spectral index around $\sim-2.8$. The typical picture of the full cascade assumes an energy transfer toward small scales mainly made by quasi-2D Alfvénic fluctuations in the MHD range (Matthaeus \& Goldstein 1982; Bieber et al. 1996) and by a mixture of dispersive modes in the ion kinetic range (Stawicki et al. 2001; Galtier \& Bhattacharjee 2003; Cho \& Lazarian 2004; Howes et al. 2008; Schekochihin et al. 2009; Boldyrev \& Perez 2012; Boldyrev et al. 2013), corresponding to local nonlinearities in Fourier space. However, embedded in this dynamics is the interaction of coherent structures where nonlinear interactions are rather local in real space: vortices, current sheets, and magnetic and flow shears are seen as the birthplace of the intermittent behavior of the turbulencewhere "dissipation" is thought to be partially, but not completely, localized (Zhdankin et al. 2013; Osman et al. 2014; Servidio et al. 2015; Wan et al. 2015; Navarro et al. 2016). The disruption of current sheets via magnetic reconnection is not only efficient in heating and accelerating particles (e.g., Loureiro et al. 2013), but also in creating ion-scale coherent structures (e.g., Greco et al. 2016), which, together with kinetic instabilities (Hellinger et al. 2015, 2017), can enhance electromagnetic fluctuations and shape the power spectrum around the ion scales. Such fluctuations can speed up the energy transfer rate and can also directly contribute to the formation of the spectral break and of the sub-ion-scale spectrum (Franci et al. 2016; Cerri \& Califano 2017). Indeed, we believe that coherent structures do play an active role in characterizing the turbulent path.

The study on turbulent reconnection dates back to the seminal work of Matthaeus \& Lamkin (1986), and mainly focuses on magnetohydrodynamics aspects (e.g., Lazarian \& Vishniac 2009; Lapenta \& Bettarini 2011; Servidio et al. 2011; Eyink 2015; Lazarian et al. 2015; Boldyrev \& Loureiro 2017; Mallet et al. 2017a). Only in the past few years has the improved numerical resources and techniques allowed for studying the interplay between turbulence and reconnection in collisionless plasma (e.g., Burgess et al. 2016; Cerri \& Califano 2017; Pucci et al. 2017). Recently, the idea that turbulence at kinetic scales is mediated by magnetic reconnection has also been supported theoretically (Loureiro \& Boldyrev 2017; Mallet et al. 2017b).

In this Letter, by means of high-resolution kinetic-hybrid Lagrangian and Eulerian simulations, we provide numerical evidence that magnetic reconnection can act as a driver for the onset of the sub-ion turbulent cascade. Following the formation of the turbulent spectrum, we show that the power-law kinetic spectrum is formed as soon as magnetic reconnection starts occurring in current sheets, independently from the existence of a fully developed spectrum at MHD scales. Such a result does not depend on the numerical approach and on the method 
adopted to drive the turbulent dynamics (forced or decaying turbulence).

Based on our analysis, we conjecture that once the sub-ion spectrum is settled down and reaches a stationary power-law regime, reconnection still remains an important energy channel feeding the small-scale turbulence.

\section{Simulation Setup}

Our model integrates the Vlasov-Maxwell equations in the hybrid approximation, where fully kinetic ions are coupled to a neutralizing massless electron background and quasi-neutrality is assumed (Winske 1985; Matthews 1994; Valentini et al. 2007). We present two direct numerical simulations employing different approaches, both in the numerical method used to integrate the Vlasov equation and in the way to achieve the turbulent state: (i) freely decaying fluctuations with the Lagrangian hybrid particlein-cell (HPIC) code CAMELIA and (ii) continuously driven fluctuations by an external low-amplitude forcing with the Eulerian hybrid Vlasov-Maxwell (HVM) code. In both codes, the ion inertial length, $d_{i}$, and the inverse ion gyrofrequency, $\Omega_{i}^{-1}$, are used as the characteristic spatial and temporal units, respectively. Both simulations are $2 \mathrm{D} 3 \mathrm{~V}$, i.e., $2 \mathrm{D}$ in real space and $3 \mathrm{D}$ in velocity space, with a uniform out-of-plane mean magnetic field. We will refer to "perpendicular" and "parallel" field components with respect to the direction of such a background field. We consider the same plasma beta for ions and electrons, $\beta_{\mathrm{i}}=\beta_{\mathrm{e}}=1$, with isothermal electrons and no initial ion temperature anisotropy. In both simulations, the energycontaining scales $\left(k_{\perp} d_{\mathrm{i}} \lesssim 0.3\right)$ and the scales significantly affected by numerical effects $\left(k_{\perp} d_{\mathrm{i}} \gtrsim 10\right)$ are basically the same. The HPIC simulation employs freely decaying, largeamplitude initial magnetic and velocity perturbations, purely perpendicular to the mean magnetic field (Franci et al. 2015a, 2015b). The HVM simulation employs instead a 3D smallamplitude initial magnetic perturbation with no velocity counterpart, fed by a continuous external injection of compressible fluctuations (Cerri et al. 2016). The grid size is $256 d_{\mathrm{i}}$ for the HPIC and $20 \pi d_{\mathrm{i}}$ for the HVM with $2048^{2}$ and $1024^{2}$ uniformly distributed grid points, respectively. The HPIC run employs 64,000 particles-per-cell, while the HVM run employs $51^{3}$ points in the velocity domain. Energy accumulation at the smallest scales is prevented by an explicit resistivity in the HPIC, empirically fine-tuned (Franci et al. 2015a), and by numerical filters in the HVM. As a consequence, both settings are representative of a "semicollisional" regime, where magnetic reconnection is enabled by resistivity and by numerical dissipation, respectively. For further details on the two numerical methods and the initial conditions see Cerri et al. (2017).

\section{Results}

As outlined by early MHD and, more recently, by kinetic simulations, an intrinsic feature of magnetized plasma turbulence is the formation of current sheets between large-scale eddies and their subsequent disruption via magnetic reconnection, generating a variety of small-scale structures and fluctuations (Matthaeus \& Lamkin 1986; Biskamp 2003; Karimabadi et al. 2013; Franci et al. 2015a; Cerri \& Califano 2017). The rms value of the current density, $|\boldsymbol{J}|$, represents a good marker of the turbulent activity (Mininni \& Pouquet 2009). The time evolution of $\operatorname{rms}(|\boldsymbol{J}|)$ (Figure 1(a)) is quite different in the two

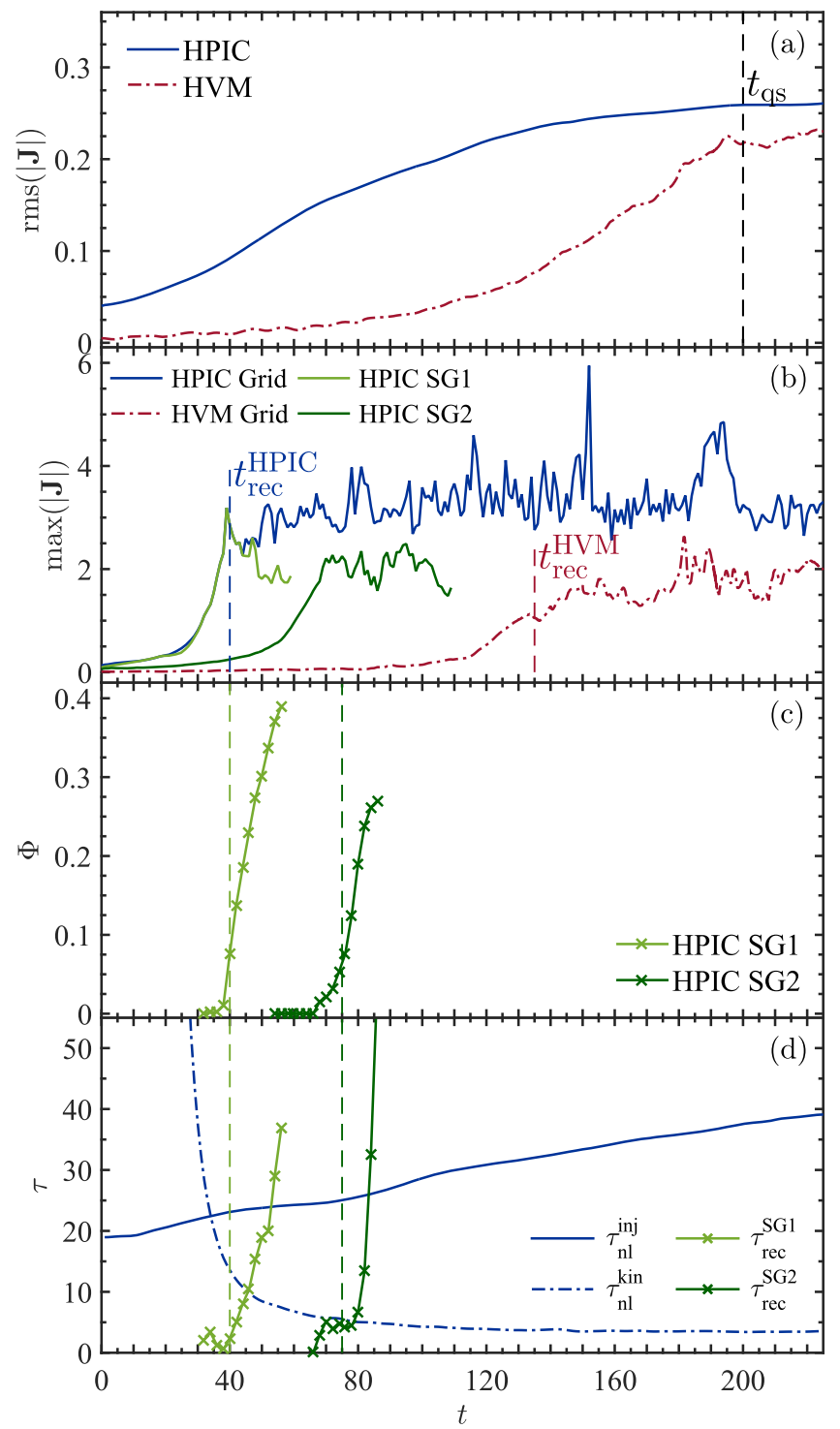

Figure 1. Time evolution of a few global and local quantities. Panel (a): $\operatorname{rms}(|\boldsymbol{J}|)$ in the HPIC (blue) and the HVM (red) runs. The black line marks the quasi-steady state at $t_{\mathrm{qs}} \sim 200$. Panel (b): $\max (|\boldsymbol{J}|)$ in the whole HPIC (blue) and HVM (red) grids, and in two HPIC subgrids (cf. Figures 2(c)-(f)). The blue and red vertical lines mark the time when magnetic reconnection start occurring, $t_{\mathrm{rec}}^{\mathrm{HPIC}}$ and $t_{\mathrm{rec}}^{\mathrm{HVM}}$, respectively. Panel (c): reconnected flux, $\Phi$, in the two HPIC grids. Panel (d): comparison between the eddy turnover time at the injection scale, $\tau_{\mathrm{nl}}^{\mathrm{inj}}$, and at kinetic scale, $\tau_{\mathrm{nl}}^{\mathrm{kin}}$, and the inverse reconnection rate in the two HPIC grids, $\tau_{\text {rec }}^{\mathrm{SG} 1}$ and $\tau_{\text {rec }}^{\mathrm{SG} 2}$.

simulations at early times, due to the different initial conditions: in HPIC, the relatively large initial fluctuations quickly drive the system toward a strong turbulent regime and rapidly generate many current sheets, resulting in an increase of $\operatorname{rms}(|\boldsymbol{J}|)$. In HVM, the turbulent dynamics is reached later, thanks to the continuous injection of momentum, but still $\operatorname{rms}(|\boldsymbol{J}|)$ starts to grow when the current sheet formation phase begins. In both cases, however, the growth saturates at $t_{\mathrm{qs}} \sim 200$, followed by a plateau. The time evolution of the maximum of $|\boldsymbol{J}|$ (Figure 1(b)) is coherent with the rms time history and can be used as a proxy for reconnection events. In both simulations, $\max (|\boldsymbol{J}|)$ is very small at early times and then rapidly increases, exhibiting a series of peaks; such maxima correspond to the evolution of current sheets that, once formed, shrink down toward a critical 
width of the order of the ion inertial length (Franci et al. 2016) before they start to reconnect, generating chains of magnetic islands (O-points; (Cerri \& Califano 2017) and locally reducing the current density intensity. To provide further evidence of this mechanism, we compute the local maxima in two HPIC subgrids, where only one intense current sheet is present. The corresponding evolution of $\max (|\boldsymbol{J}|)$ confirms what expected: $\max (|\boldsymbol{J}|)$ is initially very small and then quickly increases, reaching a local maximum after which it suddenly relaxes. Moreover, Figure 1(c) shows the reconnected flux, i.e., the difference between the out-of-plane vector potential $A_{\|}$at one of the O-points and its nearest X-point, $\Phi=A_{\|}^{\mathrm{O}}-A_{\|}^{\mathrm{X}}$, for the two HPIC subgrids mentioned above. In both cases, $\Phi$ increases very rapidly just before the local maxima of $\max (|\boldsymbol{J}|)$. Based on such an analysis, we define $t_{\text {rec }}^{\mathrm{HPIC}} \sim 40$ as the time from which reconnection is dynamically active in the HPIC case, and similarly $t_{\mathrm{rec}}^{\mathrm{HVM}} \sim 135$ for the HVM case. Note that $t_{\mathrm{rec}}^{\mathrm{HPIC}}$ and $t_{\mathrm{rec}}^{\mathrm{HVM}}$ are comparable with the initial eddy turnover time at the injection scale, which is $\tau_{\mathrm{nl}}^{\mathrm{inj}} \sim 20$ and $\sim 120$ for HPIC and HVM, respectively. This is compatible with the fact that the formation and shrinking of the first current sheets is due to the dynamics of the largest-scale eddies. Such nonlinear times have been estimated as $\tau_{\mathrm{nl}}^{\mathrm{inj}}=\left(k_{\perp}^{\mathrm{inj}} u_{i}^{\mathrm{inj}}\right)^{-1} \approx\left(k_{\perp}^{\mathrm{inj}} B^{\mathrm{inj}}\right)^{-1}$, where the ion bulk velocity fluctuations, $\boldsymbol{u}_{\mathrm{i}}$, and the magnetic fluctuations, $\boldsymbol{B}$, have been evaluated at $k_{\perp}^{\text {inj }} d_{i}=0.15$.

A qualitative view of the current sheet disruption is obtained by comparing the out-of-plane current density, $\boldsymbol{J}_{\|}$, before and after the first reconnection events occur in the whole HPIC grid (Figures 2(a)-(b)). The only difference is that some current sheets have shrunk and grown in intensity, and reconnection has occurred somewhere, generating X-points and magnetic islands, without significant changes at large scales. This process is highlighted by focusing on local changes in $\boldsymbol{J}_{\|}$and in the isocontours of $A_{\|}$in correspondence with an early (Figures 2(c)-(d); $t=[35,45])$ and a late reconnection event ((e)-(f); $t=[70,80])$ corresponding to local maxima of $\max (|\boldsymbol{J}|)$ (see Figure 1(b)).

We now focus on the effects of magnetic reconnection processes on the spectral properties. We first look at the power spectra of the parallel magnetic fluctuations, $\boldsymbol{B}_{\|}$, for the HPIC simulation (Figure 3(a), top). At $t=35$, before reconnection has occurred, no clear power law is observed, even at MHD scales. Soon after the first reconnection event $(t \sim 45)$, a power law develops at sub-ion scales, with a spectral index of $\sim-2.8$. This value is typically observed in density and parallel magnetic field in 2D simulations (Franci et al. 2015b), regardless of the plasma beta (Franci et al. 2016). At later times, the level of fluctuations in the kinetic range gradually increases, keeping the same slope.

A similar evolution is observed for the total magnetic fluctuations, $\boldsymbol{B}$ (Figure 3(a), bottom), with two main differences: (i) the asymptotic slope at sub-ion scales is steeper, around -3 , and attained gradually, later than the first reconnection event; (ii) the MHD part of the spectrum continues to flatten slowly, until a $-5 / 3$ power law develops, much later. This behavior is consistent with a picture where reconnection drives a kinetic-scale turbulent cascade. Since only perpendicular magnetic fluctuations were imposed as an initial condition, the reconnection-driven contribution is better appreciated in the $\boldsymbol{B}_{\|}$fluctuations (as well as in the density fluctuations; not shown here). Only later, the direct cascade from larger scales bring its contribution to the total magnetic power spectrum, due to the Alfvénic-like $\boldsymbol{B}_{\perp}$ component. The time required for the formation of a stable and extended power law in the kinetic range, once reconnection has started occurring at $t \sim 40$, is $\sim 5$, i.e., shorter than the eddy turnover time, $\tau_{\text {inj }}^{\text {nl }} \gtrsim 20$. Moreover, Figure 1(d) shows that the inverse reconnection rate of the first event, $\tau_{\mathrm{rec}}^{\mathrm{SG} 1}$, is indeed smaller than the eddy turnover time estimated at both the injection scale, $\tau_{\mathrm{nl}}^{\mathrm{inj}}$, and at kinetic scales, $\tau_{\mathrm{nl}}^{\mathrm{kin}}$, at the time when the reconnected flux starts increasing. The latter is given by $\tau_{\mathrm{nl}}^{\mathrm{kin}}=\left(k_{\perp}^{\mathrm{inj}} \boldsymbol{u}_{\mathrm{e}}^{\mathrm{inj}}\right)^{-1} \approx\left(k_{\perp}^{\mathrm{inj} 2} \boldsymbol{B}^{\mathrm{inj}}\right)^{-1}$, where $\boldsymbol{u}_{\mathrm{e}}$ is the electron bulk velocity, and we chose the scale $k_{\perp} d_{i}=2$, which will later correspond to the spectral break. The comparison of $\tau_{\text {rec }}^{\mathrm{SG} 1}$ with $\tau_{\mathrm{nl}}^{\mathrm{inj}}$ and $\tau_{\mathrm{nl}}^{\mathrm{kin}}$ indicates that reconnection is indeed very efficient in transferring energy at kinetic scales, faster than a direct cascade from the injection scale, causing a strong and rapid decrease of $\tau_{\mathrm{nl}}^{\text {kin }}$ (see Figure 1(d)) in correspondence with the first local maximum of $\max (|\boldsymbol{J}|$ ) (see Figure 1(b)) and the sudden increase of $\Phi$ (see Figure 1(c)).

This analysis, together with the evidence that a kinetic cascade forms rapidly and despite the absence of a Kolmogorov-like cascade at large scales, suggests that the kinetic spectrum is not a simple extension of the MHD spectrum through a "classic" cascade involving only local interactions in $k$-space. Energy is directly injected at small scales via non-local interactions in Fourier space mediated by magnetic reconnection occurring in strong and thin current sheets, whose width is of the order of the ion scales (e.g., Franci et al. 2016). Reconnection events produce ion-scale magnetic islands, which can generate an inverse flux toward larger scales by merging and/or can start a transfer of energy toward smaller scales. The net result is a direct cascade at sub-ion scales. This channel for the generation of the magnetic field spectrum at sub-ion scales is sketched in Figure 4. Concurrently, although on larger timescales, a direct turbulent cascade develops from the largest scales, generating eddies of smaller and smaller sizes, which interact and form many other current sheets, injecting additional energy at ion scales. This mechanism can be appreciated by looking at the evolution of magnetic fluctuations (see Figures $2(\mathrm{k})-(\mathrm{m})$ and the corresponding animations).

Let us now consider the magnetic field spectra of the forced HVM simulation (Figure 3(b)). Initially, the energy at large scales grows slowly, due to the external forcing, and develops into a Kolmogorov-like cascade at $t \sim 120$, while no significant power is present at kinetic scales yet. Later, once the large-scale fluctuations reach roughly the same level as in the HPIC case, reconnection starts occurring (at $t_{\mathrm{rec}}^{\mathrm{HVM}} \sim 135$; see Figures $2(\mathrm{~g})-(\mathrm{j}))$, and a power-law spectrum forms also at kinetic scales, with the same spectral index of -2.8 in $\boldsymbol{B}_{\|}$. Finally, a stationary regime is reached, characterized by a double power-law behavior, in agreement with the HPIC simulation (Cerri et al. 2017).

\section{Conclusions}

In the present work, we have provided the first numerical evidence that a sub-ion-scale cascade in collisionless plasmas can develop independently from a Kolmogorov-like cascade at MHD scales, triggered by magnetic reconnection. This new picture of the turbulent dynamics across the ion break has been achieved by analyzing two high-resolution hybrid simulations, which employ different methods to drive the turbulence and different numerical methodologies to simulate the system 

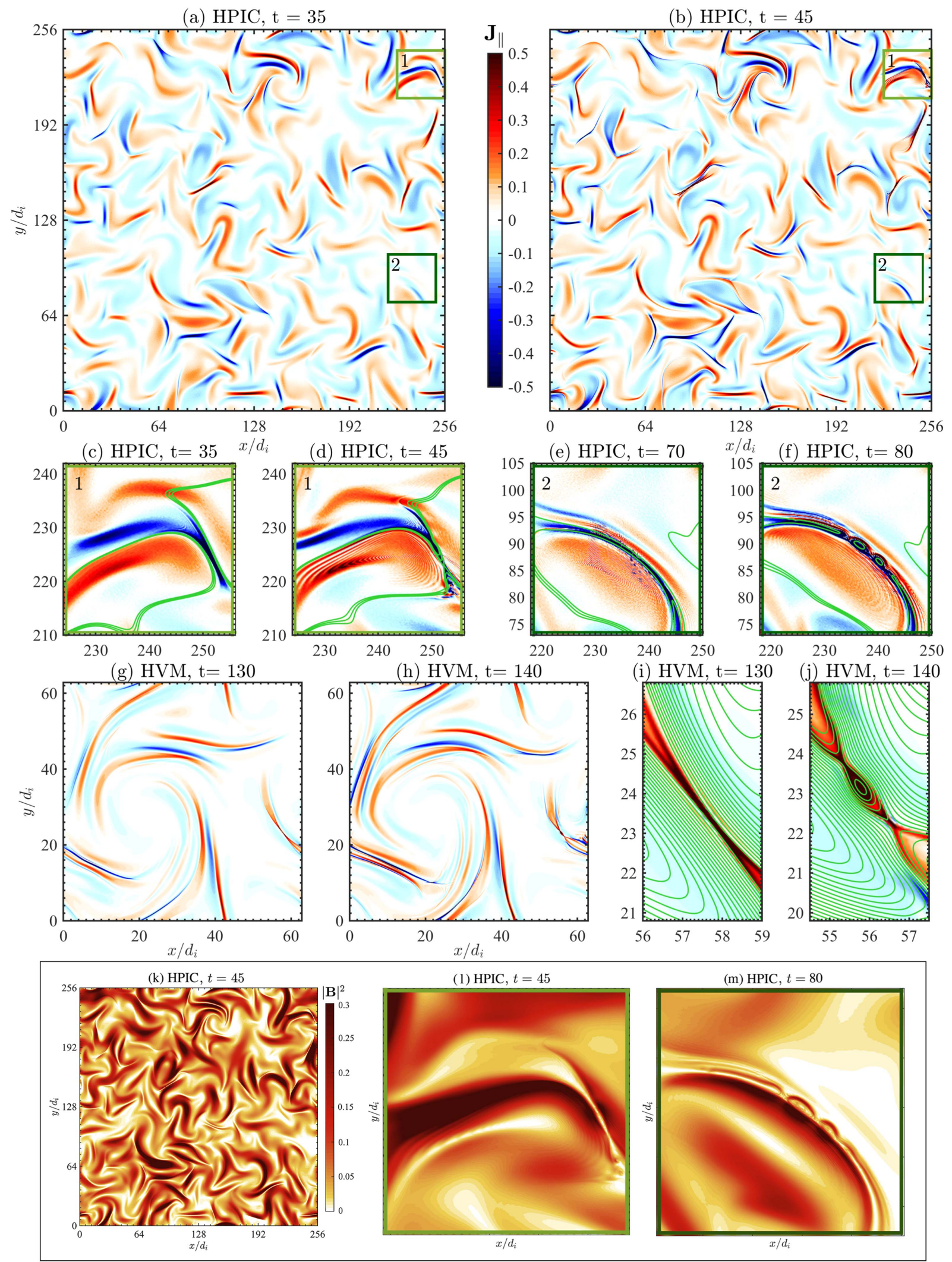

Figure 2. Contours of the out-of-plane current density, $\boldsymbol{J}_{\|}$, before and after the onset of magnetic reconnection. Panels (a)-(b): whole HPIC grid around $t_{\text {rec }}^{\text {HPIC }} \sim 40$. Panels (c)-(f): two HPIC subgrids, containing one reconnecting current sheet. Additionally, isocontours of $A_{\|}$are drawn in green. Panels (g)-(j): the same for the HVM simulation, but around $t_{\text {rec }}^{\mathrm{HVM}} \sim 135$. Panels $(\mathrm{k})-(\mathrm{m})$ : contours of $|\boldsymbol{B}|^{2}$ for the whole HPIC grid and the two HPIC subgrids.

(Animations $(\mathrm{k}, 1$, and $\mathrm{m})$ of this figure are available.) 
(a)

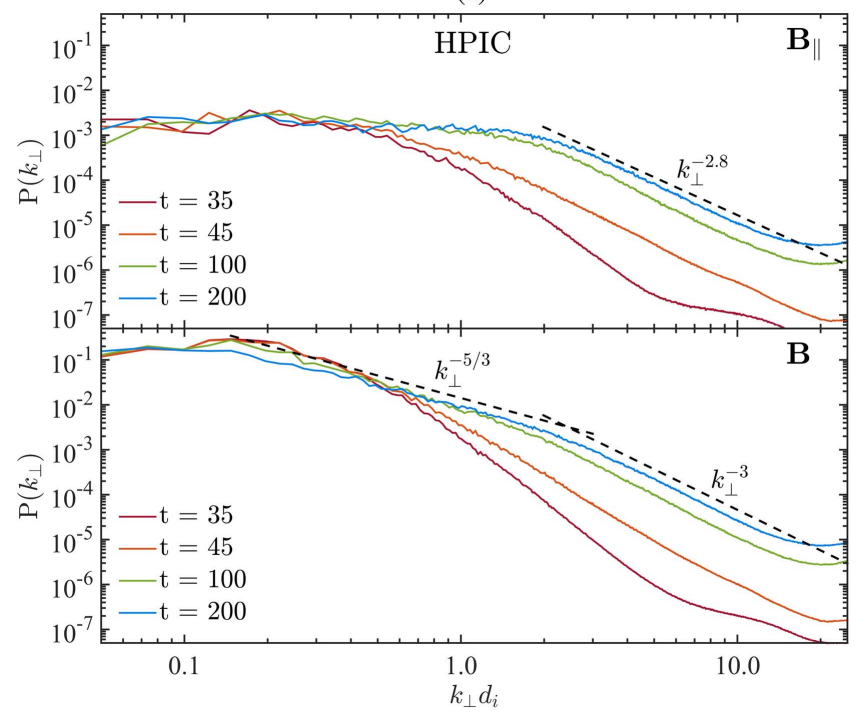

(b)

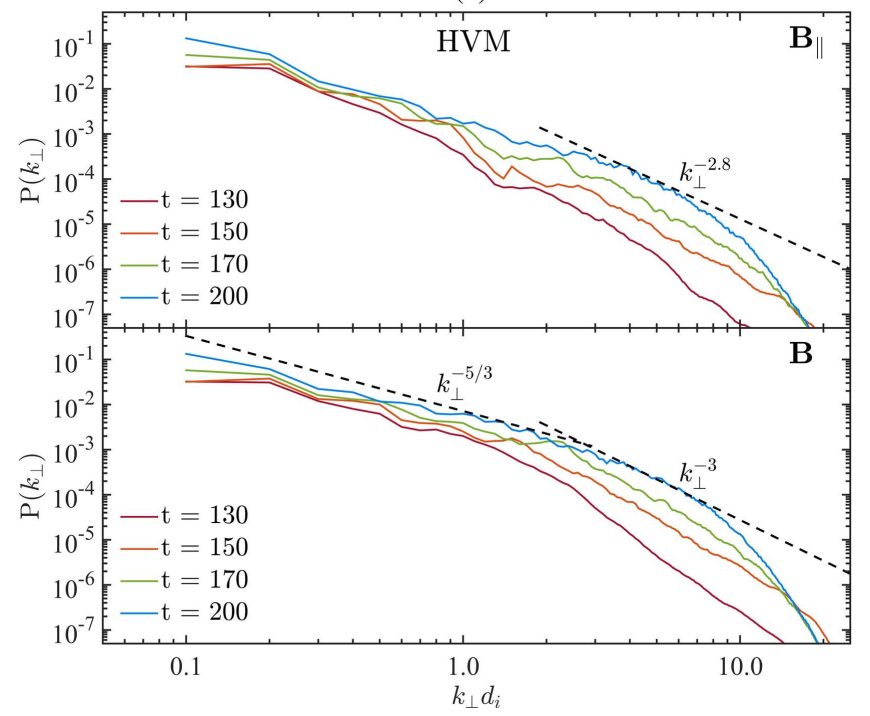

Figure 3. (a) Power spectra of the parallel (top) and total (bottom) magnetic fluctuations for the HPIC run before (red) and after (orange) the first magnetic reconnection events occur, at an intermediate time (green) and when the quasisteady state is reached (light blue). Characteristic power laws are drawn as a reference. (b) Same as in panel (a), but for the HVM run.

evolution, a Lagrangian HPIC, and an Eulerian hybrid VlasovMaxwell approach.

In HPIC, an extended power law in the spectrum of the parallel magnetic fluctuations forms early at sub-ion scales, without a well-developed turbulent spectrum at MHD scales. Only later, a Kolmogorov-like cascade for the (total) magnetic fluctuations gradually develops in the MHD range, due to the contribution of the perpendicular components. In HVM, conversely, the same power law at kinetic scales is achieved after a Kolmogorov-like MHD cascade is established but, again, only as soon as the first reconnection event has occurred. In both cases, a fully developed turbulence state is achieved, in which both the MHD and the sub-ion spectral slopes are quasistationary and attain the values of $-5 / 3$ and -3 , respectively. Furthermore, the kinetic range exhibits the same properties in the two cases (comparable level of parallel and perpendicular

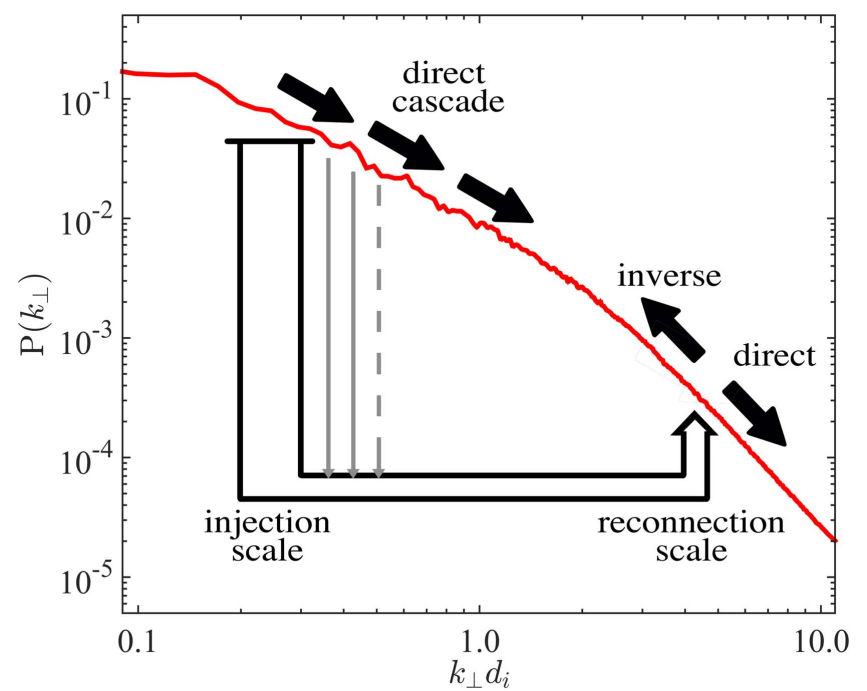

Figure 4. Schematic view of the development of the turbulent cascade as a combination of two mechanisms: (i) direct cascade through local transfers in Fourier space; (ii) injection of energy from large-scale vortices directly into small-scale structures via reconnection, local in real space but non-local in Fourier space.

fluctuations; see Cerri et al. 2017), despite the quite different MHD-range behavior: in HPIC, the cascade is carried by perpendicular fluctuations, while in HVM the parallel component dominates.

The correspondence between the onset of magnetic reconnection events and the formation of a stable power-law spectrum at kinetic scales, together with the fact that the inverse reconnection rate is initially much shorter than the eddy turnover time around ion scales, is clear evidence that in both simulations the former acts as a trigger for the latter. The present analysis does not allow us to determine whether or not reconnection still remains the main energy source feeding the small-scale turbulence once a stationary state is reached. Nevertheless, the interaction between large-scale eddies keeps driving the formation of many, randomly distributed, current sheets. When these undergo reconnection, their width, intensity, and reconnection rate are still of the same order of the early events. Although we are not able to quantitatively evaluate what fraction of the turbulent cascade is driven by spatially localized reconnection events, we conjecture that reconnection is likely the preferred/fastest path for energy injection at ion scales, based on the fact that: (i) the local maxima of $\max (|\boldsymbol{J}|)$, directly linked to reconnection events, exhibit approximately the same intensity from $t_{\text {rec }}$ on, indicating that strong current sheets keep forming and disrupting; (ii) $\tau_{\mathrm{nl}}^{\mathrm{kin}}$ rapidly decreases as soon as reconnection begins, adjusting and settling to an asymptotic value of $\tau_{\mathrm{nl}}^{\mathrm{kin}} \lesssim 5 \Omega_{i}^{-1}$, which is comparable to the inverse reconnection rate $\tau_{\text {rec }}$; and (iii) once formed, the power law at kinetic scales is well maintained and the spectra only grow in amplitude until the quasi-stationary state is reached, indicating that the number of reconnecting current sheets increases until a balance between formation and disruption is achieved. We thus suggest that any theory of the turbulence cascade down to ion scales should carefully take into account the role of the magnetic reconnection, which should not be seen only as the location where 
dissipative effects are dominant (e.g., Loureiro \& Boldyrev 2017; Mallet et al. 2017b).

Determining how important this process is in real turbulence will require further investigations by means of high-resolution 3D simulations. Indeed, 3D MHD (e.g., Landi et al. 2008) and kinetic (e.g., Gingell et al. 2015) instabilities can significantly affect the nonlinear evolution of reconnection in current sheets, possibly limiting the energy transfer toward smaller scales and also determining the structures of its byproducts: the flux ropes observed in our 2D simulations (see the animations associated with Figures 2(k)-(m)) and predicted by Mallet et al. (2017a) could in principle be completely destroyed in a strong turbulent medium $\left(\delta b / B_{0} \gtrsim 1\right)$. In this regard, Franci et al. (2017) shows that magnetic and current structures are more complex in $3 \mathrm{D}$, but their spectral properties at kinetic scales look almost identical. Although, in this case, the identification of reconnection sites is not straightforward, a preliminary analysis supports our findings (e.g., a correspondence between the first peak in the maximum of the current and the development of the kinetic power law is observed).

The authors wish to acknowledge valuable discussions with D. Burgess, T. Horbury, C. H. K. Chen, J. E. Stawarz, and F. Pegoraro. S.C.C. and F.C. thank F. Rincon for the implementation of the external forcing and C. Cavazzoni (CINECA, Italy) for his essential contribution to the HVM code parallelization and performances. L.F. is funded by Fondazione Cassa di Risparmio di Firenze, through the project "Giovani Ricercatori Protagonisti." L.M. was funded by the UK STFC grant ST/N000692/1. P.H. acknowledges GACR grant 15-10057S. The authors acknowledge PRACE for awarding access to resource Cartesius based in the Netherlands at SURFsara through the DECI-13 (Distributed European Computing Initiative) call (project HybTurb3D), CINECA for awarding access to HPC resources under the ISCRA initiative (grants HP10BUUOJM, HP10BEANCY, HP10B2DRR4, HP10CGW8SW, HP10C04BTP), and the Max Planck Computing and Data Facility (MPCDF) in Garching (Germany).

\section{ORCID iDs}

Luca Franci (1) https://orcid.org/0000-0002-7419-0527

Silvio Sergio Cerri (1) https://orcid.org/0000-0003-0562-6574

Francesco Califano (it) https://orcid.org/0000-0002-9626-4371

Simone Landi 1 https://orcid.org/0000-0002-1322-8712

Emanuele Papini (i) https://orcid.org/0000-0002-7969-7415

Andrea Verdini (1) https://orcid.org/0000-0003-4380-4837

Lorenzo Matteini (1) https://orcid.org/0000-0002-6276-7771

Frank Jenko iㅏ https://orcid.org/0000-0001-6686-1469

Petr Hellinger (1) https://orcid.org/0000-0002-5608-0834

\section{References}

Alexandrova, O., Saur, J., Lacombe, C., et al. 2009, PhRvL, 103, 165003 Bale, S. D., Kellogg, P. J., Mozer, F. S., Horbury, T. S., \& Reme, H. 2005, PhRvL, 94, 215002
Bieber, J. W., Wanner, W., \& Matthaeus, W. H. 1996, JGR, 101, 2511

Biskamp, D. 2003, in Magnetohydrodynamic Turbulence (Cambridge: Cambridge Univ. Press), 310

Boldyrev, S., Horaites, K., Xia, Q., \& Perez, J. C. 2013, ApJ, 777, 41

Boldyrev, S., \& Loureiro, N. F. 2017, ApJ, 844, 125

Boldyrev, S., \& Perez, J. C. 2012, ApJL, 758, L44

Bruno, R., \& Carbone, V. 2013, LRSP, 10, 2

Burgess, D., Gingell, P. W., \& Matteini, L. 2016, ApJ, 822, 38

Cerri, S., Franci, L., Califano, F., Landi, S., \& Hellinger, P. 2017, JPIPh, 83, 705830202

Cerri, S. S., \& Califano, F. 2017, NJPh, 19, 025007

Cerri, S. S., Califano, F., Jenko, F., Told, D., \& Rincon, F. 2016, ApJL, 822, L12

Chen, C. H. K., Horbury, T. S., Schekochihin, A. A., et al. 2010, PhRvL, 104, 255002

Cho, J., \& Lazarian, A. 2004, arXiv:astro-ph/0411031

Eyink, G. L. 2015, ApJ, 807, 137

Franci, L., Landi, S., Matteini, L., Verdini, A., \& Hellinger, P. 2015a, ApJ, 812,21

Franci, L., Landi, S., Matteini, L., Verdini, A., \& Hellinger, P. 2016, ApJ, 833, 91

Franci, L., Landi, S., Verdini, A., Matteini, L., \& Hellinger, P. 2017, ApJ, submitted (arXiv:1711.02664)

Franci, L., Verdini, A., Matteini, L., Landi, S., \& Hellinger, P. 2015b, ApJL, 804, L39

Galtier, S., \& Bhattacharjee, A. 2003, PhPl, 10, 3065

Gingell, P. W., Burgess, D., \& Matteini, L. 2015, ApJ, 802, 4

Greco, A., Perri, S., Servidio, S., Yordanova, E., \& Veltri, P. 2016, ApJL, 823, L39

Hellinger, P., Landi, S., Matteini, L., Verdini, A., \& Franci, L. 2017, ApJ, 838,158

Hellinger, P., Matteini, L., Landi, S., et al. 2015, ApJL, 811, L32

Howes, G. G., Cowley, S. C., Dorland, W., et al. 2008, JGR, 113, A05103

Karimabadi, H., Roytershteyn, V., Wan, M., et al. 2013, PhPl, 20, 012303

Landi, S., Londrillo, P., Velli, M., \& Bettarini, L. 2008, PhPl, 15, 012302

Lapenta, G., \& Bettarini, L. 2011, EL, 93, 65001

Lazarian, A., Eyink, G., Vishniac, E., \& Kowal, G. 2015, RSPTA, 373, 20140144

Lazarian, A., \& Vishniac, E. T. 2009, RMxAC, 36, 81

Loureiro, N. F., \& Boldyrev, S. 2017, arXiv:1707.05899

Loureiro, N. F., Schekochihin, A. A., \& Zocco, A. 2013, PhRvL, 111, 025002

Mallet, A., Schekochihin, A. A., \& Chandran, B. D. G. 2017a, MNRAS, 468, 4862

Mallet, A., Schekochihin, A. A., \& Chandran, B. D. G. 2017b, arXiv:1707. 05907

Matteini, L., Alexandrova, O., Chen, C. H. K., \& Lacombe, C. 2017, MNRAS, 466, 945

Matthaeus, W. H., \& Goldstein, M. L. 1982, JGR, 87, 6011

Matthaeus, W. H., \& Lamkin, S. L. 1986, PhFl, 29, 2513

Matthews, A. P. 1994, JCoPh, 112, 102

Mininni, P. D., \& Pouquet, A. 2009, PhRvE, 80, 025401

Navarro, A. B., Teaca, B., Told, D., et al. 2016, PhRvL, 117, 245101

Osman, K. T., Matthaeus, W. H., Gosling, J. T., et al. 2014, PhRvL, 112, 215002

Pucci, F., Servidio, S., Sorriso-Valvo, L., et al. 2017, ApJ, 841, 60

Sahraoui, F., Goldstein, M. L., Belmont, G., Canu, P., \& Rezeau, L. 2010, PhRvL, 105, 131101

Schekochihin, A. A., Cowley, S. C., Dorland, W., et al. 2009, ApJS, 182, 310 Servidio, S., Valentini, F., Perrone, D., et al. 2015, JPIPh, 81, 325810107

Servidio, S., Dmitruk, P., Greco, A., et al. 2011, NPGeo, 18, 675

Stawarz, J. E., Eriksson, S., Wilder, F. D., et al. 2016, JGR, 121, 11

Stawicki, O., Gary, S. P., \& Li, H. 2001, JGR, 106, 8273

Valentini, F., Trávníček, P., Califano, F., Hellinger, P., \& Mangeney, A. 2007, $\mathrm{JCoPh}, 225,753$

Wan, M., Matthaeus, W. H., Roytershteyn, V., et al. 2015, PhRvL, 114 , 175002

Winske, D. 1985, SSRv, 42, 53

Zhdankin, V., Uzdensky, D. A., Perez, J. C., \& Boldyrev, S. 2013, ApJ, 771,124 